\title{
Much more than elegant
}

In the words of the immortal Fats Waller, "If this ain't love, it'll have to do until the real thing comes along." So while the publication last month of the completed genome for the multicelluar soil worm, Caenorhabditis elegans, may be comparable to "only finishing a section of the transcontinental railroad," as Geoffrey Duyk, the chief scientist at Exelixis Pharmaceuticals, Inc., a South San Francisco genomics company, described to us, it was also a watershed moment for this newest cornerstone of the biotechnology industry, and cause as he put it "for a bit of a coming out party." For poised, now more translucent than transparent, between the relatively tractable bacterial and yeast genomes and the more dauntingly complex ones of the fly, mouse, and human, the C. elegan's chromosome set is expected to provide a powerful new array of tools with which to produce the pharmacueticals, enzymes, screens, and other promised products of genomics-based biotechnology.

\section{Brenner's model and genomic modeling}

When Sydney Brenner turned his attention, 30 years ago, to C. elegans as the perfect organism with which to model the "functional genetics" of multicellularity, he did so with extremely good reasons, as the work from his and a whole generation of worm molecular biology labs has more than demonstrated. Not only does this wonderfully transparent nematode replicate quickly (a few days), its almost 1000 cells reproduce many of the patterns and interactions of much larger and more complex animals, including a fundamental nervous sytem that contains almost all the biochemical components of the vertebrate one. But back then, Brenner also suspected that the genetic constitution and organization of C. elegans might serve as a model for other animals. And apparently it does, as estimates of gene conservation between nematodes and mice, for example, are put at $+90 \%$ and a figure of $70 \%$ is given for worms and humans. Indeed, the information available now about C. elegans' 12 chromosomes, containing 97 megabases of DNA and encoding almost 20,000 proteins (one-fifth perhaps of a human complement), has already prompted the prediction of an accelerated completion date of 2003 for the Human Genome Project.

It is this combination of experimental accessibility (including now all the tricks of GFP and iRNA), complete genetic and a wealth of functional information-perhaps more important than gene conservation per se, is the conservation of metabolic pathways involving the products of those of genes-thanks to work from Robert Horvitz, John Sulston, and others who have mapped the genetic wiring of many of its developmental programs, that makes C. elegans so potentially useful in genomic modeling research-and eventually the development of novel pharmaceuticals. By comparison, the variables and interactions of human, or even mouse genetics, are complete terras incognitas when it comes to locating specific targets or aberrant disease-producing biochemistries.

\section{The road ahead}

But this transformation will not happen overnight, nor will it necessarily be straightforward. The presenilin genes of C. elegans obviously do not cause Alzheimer's in worms. For the moment, and immediate future, the central focus of pharmaceutical genomic research is on uncovering the basic reactions that make up potential pathophysiological pathways, though their precise biochemistries may diverge and their phenotypes in their respective organisms differ.

Daf-16 signaling, for example, has been implicated as a key feature of diabetes, but the daf16 product itself makes a poor potential drug target. Investigating this pathway in, and using information deduced from, $C$. elegans will be invaluable in translating this research finding into a new treatment. Moreover, because C. elegans is a member of a family that includes many important human, plant, and animal pathogens, the newest addition to the rich constellation of data and information available about this worm will have a dramatic and quite likely rapid inpact even without recourse to phylogenetic and distant homology arguments.

Finally, in joining the chorus of those praising this latest triumph of our new global genetics, we also need to note the bioinformatic accomplishment that accompanied the progress of the worm genome project.

Introduction of the ACDB database system to present and disseminate genomic data to the entire C. elegans research community has had an inpact likened by one genomic's company executive to that of the first computer spreadsheets in business.

So for these and many other worthwhile endeavors that have and will benefit from the accomplishment of Sulston's and Waterson's teams, we take this New Year opportunity to say to them, on behalf of ourselves and our readers, thank you very much indeed. 